BBAEXP 92357

\title{
High-resolution analysis of c-fos chromatin accessibility using a novel DNase I-PCR assay
}

\author{
Junli Feng ${ }^{b, 1}$ and Bryant Villeponteau ${ }^{\text {a,b }}$ \\ "Deparment of Biological Chemistry and" The Institute of Gerontology, University of Michigan, Ann Arbor. MI (USA)
}

(Received 14 October 1991)

Key words: Chromatin accessability: c-fos: Oncogene: DNase sensitive region

In our previous study, c-fos chromatin accessibility was assiyed using DNase I digestion and Southern blot analysis. This low-resolution mapping of c-fos cluromatin accessibility demonstrated that serum stimulation of the c-fos enhancer induces a reversible increase in c-fos DNase I sensitivity and suggested that a $5^{\prime}$ to $3^{\prime}$ gradient of DNase I sensitivity may form downstream from the c-fos enhancer. To confirm the existence of a $5^{\prime}$ to $3^{\prime}$ gradient of accessibility, we have recently developed a high-resolution polymerase chain reaction (PCR) assay for DNase I sensitivity. Using this novel DNase I assay, we have reliably detected position- and time-dependent gradients of chromatin accessibility around the c-fos cnhancer. These data confirm our earlier results and further support the hypothesis that the changes in c-fos chromatin accessibility originate near the $5^{\prime}$ enhancer. As a technique for future examinations of gene structure, our data demonstrate the value of thic DNase I-PCR assay for rapidly preparing comprehensive and high-resolution maps of chromatin accessibility for any sequenced genomic region.

\section{Introduction}

It is well established that active genes are more sensitive to DNase I digestion than inactive genes. Generalized or domain DNase I sensitivity can extend over large chromatin regions of 12 to 100 kilobases and includes nontranscribed spacer regions as well as active genes [1-4]. We have been interested in how enhanced DNase I accessibility is initiated and imposed over such large domains. If the enhanced accessibility is initiated at a regulatory site and propagates from this site, then a declining gradient of DNase I sensitivity might be observed under some circumstances owing to damping effects with distance from the origin.

Recently, we presented evidence consistent with the existence of a $5^{\prime}$ to $3^{\prime}$ gradient of DNase I sensitivity along the c-fos gene during basai transcription [5]. However, the resolution and reliability of the standard assays for DNase I sensitivity are poor, so that the existence of a gradient of DNase I sensitivity was

\footnotetext{
1 Present Address: Department of Biochemistry, Wayne State University Medical School, Detroit, MI, USA.

Abbreviations: PCR, polymerase chain reaction: $A S$, argininosuccinate synthetase: $b p$, base pair(s); $k b$, kilobase(s)

Correspondence: B. Villeponteau, Institute of Gerontology, University of Michigan, 300 North Ingalls, Ann Arbor, MI 48109-2007, USA.
}

difficult to verify using current technology. The advent of PCR technology has made it possible to design a novel assay for domain DNase I sensitivity which has a higher resolution and improved reliability.

The DNase I-PCR assay uses standard DNase I digestion and then determines the degree of DNase I cleavage at a particular genetic location $(350$ to $390 \mathrm{bp}$ in length) by PCR amplification of the region. A single cut within a probed sequence will prevent amplification of the sequence. Thus, coamplification of active and inactive regions should allow detection of the preferential sensitivity of the active domain in comparison to the inactive one. We have used this high-resolution PCR technique to refine our previous mapping of DNase I sensitivity along the c-fos gene during serum stimulation. In unstimulated cells and during the early stages of serum stimulation, the DNase I-PCR data confirm the existence of a gradient of chromatin accessibility that peaks near the $5^{\prime} \mathrm{c}$-fos enhancer.

\section{Materials and Methods}

Recombinant DNA and PCR probes. The human c-fos gene, denoted pc-fos(human)-1 [6,7], was obtained from Inder Verma as a 9 kb EcoRl insert in pBR322. A plasmid containing the argininosuccinate synthetase (AS) coding region [8] was obtained from Svend Freytag. The AS probe cross-hybridizes with inactive AS pseudogene fragments and was used as a 
probe for inactive genes in the blot analysis. The region upstream of the c-fos enhancer $(-2$ to 0 in Figs. 4 and 5) has not been sequenced, so that PCR primers from this region could not be prepared. To obtain DNase I sensitivity data on this upstream region, we have labeled a $1.4 \mathrm{~kb}$ upstream restriction fragment and probed Southern blots of DNase I digested DNA. PCR primers were prepared by the University of Michigan DNA sequencing core. All primers were $30 \mathrm{bp}$ in length and were designed to probe the middle portion of the $\beta$-globin gene ( $460 \mathrm{bp}$ long) and four c-fos regions ( $\mathrm{F} 1$ to $\mathrm{F} 4$ spanning 350 to $390 \mathrm{bp}$ ). The $c$-fos and $\beta$-globin primer sequences were: F1A5'TCTTACTACCACTCACCCGCAGACTCCTTC3'), FIB (5'CGAAGAAGCCAGGCTCTAGTTAGCGAG'TTT3'), F2A(5'GAATAAGATGGCTGCAGCCAAATGCCGCAA3'), F2B(5'CAGTCAGATCAAGGGAAGCCACAGACATCT3'), F3A(5'GGACACACCCTCTGTCTGATCCCTTATCAA3'), F3B(5' CGAGCAGGAAGATTCTAATGCCGATAACG G3'), F4A(5'TGAAGAAGGGCAGCTGGACCTTCGGACATT3'), F4B(5'GGGATTTGGAAGGAGCCATTTAGCTGGAGA3'), GlobinA(5'GGAGACCAATAGAAACTGGGCATGTGGAGA3'), GlobinB (5'CGATCCTGAGACTTCCACACTGATGCAATC3').

Cell culture and serum induction. HeLa cells were grown in monolayer cultures in a $1: 1$ mixture of Dulbecco's modified Eagle's medium and Ham's nutrient F-12 medium (Sigma, No. 8900) supplemented with bovine calf serum (Hazelton laboratories). To prepare serum-starved cells, HeLa cells were grown to $70 \%$ confluency in the above medium with $10 \%$ serum, transferred to medium with $0.5 \%$ serum for $20 \mathrm{~h}$, and finally transferred to medium with no serum for $\mathbf{3 0}$ min. To induce c-fos, medium with $15 \%$ serum was added and the cells were incubated at $37^{\circ} \mathrm{C}$ for various times before harvesting. The cells were washed, scraped from the plate and collected in ice-cold phosphatebuffered saline.

Isolation of nuclei and DNase I digestions. The cells were pelleted and resuspended in cold buffer $A$ (10 $\mathrm{mM}$ Tris- $\mathrm{HCl}, 10 \mathrm{mM} \mathrm{NaCl}, 3 \mathrm{mM} \mathrm{MgCl}_{2}$ ) along with $0.4 \%$ Nonidet P-40 and $1 \mathrm{mM} p$-(chloromercuri)benzenesulfonic acid as described previously [9]. After 5 $\mathrm{min}$, the resulting lysate was centrifuged at $2000 \times g$ for $5 \mathrm{~min}$ to pellet the nuclei. The nuclei were resuspended in buffer $A$ at a concentration of $800 \mu \mathrm{g} / \mathrm{ml}$ (assayed by dilution into $2 \mathrm{M} \mathrm{NaCl}$ and $5 \mathrm{M}$ urea) and stored on ice briefly. Five $360 \mu \mathrm{l}$ aliquots of each sample were preincubated at $37^{\circ} \mathrm{C}$ for $5 \mathrm{~min}$. The nuclei were digested for 3 min at $37^{\circ} \mathrm{C}$ with various concentrations of DNase I (Sigma, D-5025). Reactions were stopped by adding sodium dodecyl sulfate (SDS) and EDTA to final concentrations of $0.4 \%$ SDS and 20 mM EDTA.
DNA isolation, Southern blotting, hybridization and band integration. DNA was purified from the nuclei as previously described [10] and digested overnight at $37^{\circ} \mathrm{C}$ with 2 units of various restriction enzymes per $\mu \mathrm{g}$ of DNA. The restricted DNA was subjected to either PCR or Southern blot analysis. Blot analysis was carried out by standard methods [11] as previously described [5]. The blots were first hybridized to labeled c-fos probe. Following autoradiography at $-80^{\circ} \mathrm{C}$ for 5 to 10 days, the autoradiographs were scanned with a densitometer (Hoefer Scientific) and the bands were integrated using a microcomputer. The Southern blots were rehybridized to an AS probe, which cross-hybridizes with a family of nontranscribed AS pseudogenes [5]. The AS pseudogenes are used as inactive gene standards for blots, as many different sized AS fragments are labeled and all have the same DNase I sensitivity as bulk chromatin (data not shown).

RNA isolation, blotting and hybridization. Total cellular RNA was purified by guanidine-thiocyanate extraction [12], denatured with glyoxal and dimethylsulfoxide [13] and electrophoresed on a 1\% agarose gel. Transfer to nitrocellulose and subsequent hybridization were carried out using the same conditions as for Southern blots. As a check on the quality and quantity of loaded RNA, agarose gels were stained with ethidium bromide and the RNA was visualized under ultraviolet light prior to Northern blotting.

PCR analysis of DNase I treated samples. DNase I digested DNA samples $(0.5 \mu \mathrm{g})$ were analyzed using duplex PCR reactions with primers for the $\beta$-globin control and c-fos test probes. Amplification was carried out at three standard temperatures $(94,63$ and $72^{\circ} \mathrm{C}$ ) for 22 cycles on a thermocycler. Thermocycler well-to-well uniformities of less than $\pm 0.5^{\circ} \mathrm{C}$ were required in order to obtain precise quantitative results using PCR. The final reaction products were electrophoresed on a $6 \%$ polyacrylamide gel, stained with ethidium bromide, visualized under $254 \mathrm{~nm}$ ultraviolet light, and photographed with $4^{\prime \prime} \times 5^{\prime \prime}$ negative Tmax film (Kodak). Quantitation of the ethidium PCR gels was appropriately linear only if high-density Tmax film was used in place of low-density, high-contrast Polaroid film. The PCR bands in the film were quantitated by scanning with a Hoefer densitometer and the areas under the peaks were integrated with the aid of a microcomputer. Equivalent results were obtained if quantitation was carried out using radioactively-labeled nucleotides and direct-band counting by Cerenkov radiation. Normalized DNase I sensitivity values were calculated from the equation: $S_{\mathrm{F}}=\left(F_{\mathrm{U}} / G_{\mathrm{U}}\right) \div\left(F_{\mathrm{D}} /\right.$ $\left.G_{\mathrm{D}}\right) \times T$ where $F$ and $G$ are c-fos and globin band intensities for the undigested (U) or digested (D) samples and $T$ is the ratio of globin to c-fos sizes.

DNase I hypersensitive sites. DNase I hypersensitive sites were mapped by indirect-end labeling experi- 
ments as described previously [5]. The two major hypersensitive sites are shown as downward arrows in the c-fos map (Figs. 4 and 5) and occur over the c-fos enhancer and promoter. A minor hypersensitive site is also found inside the c-fos transcription unit (not shown). All PCR sensitivity probes were located at least $300 \mathrm{bp}$ from any hypersensitive site to ensure that the sensitivity probes would not be perturbed by changes in hypersensitivity.

Sensitivity of naked DNA to DNase I. To control for the possibility that some c-fos DNA sequences differ in native sensitivity to DNase I, purified HeLa DNA was subjected to limited DNase I digestion and then assayed for relative DNase I sensitivity using the PCR technique. With naked DNA, all globin and c-fos sequences were digested equally (data not shown), demonstrating that preferential c-fos DNase I sensitivity requires chromatin structure.

\section{Results}

The DNase I-PCR assay rapidly and reliably determines chromatin accessibility during $c$-fos induction

As a test for the PCR assay of DNase I sensitivity, we have analyzed DNase I sensitivity of chromatin during c-fos induction by both the standard Southern blot assay [5] and by the PCR assay. In the experiment, serum-starved HeLa cells were stimulated with serum for 0,15 or $60 \mathrm{~min}$ and nuclei were prepared from the three time points. Under these conditions, c-fos mRNA peaks at $15 \mathrm{~min}$ and falls to basal levels by $60 \mathrm{~min}$ (Fig. 1 and Refs. 5,14). The nuclei were subjected to DNase 1 digestion and the same DNase I-digested DNA was analyzed by the Southern blot assay [5] or by the new PCR assay. In the PCR assay, active and inactive DNA regions were amplified for 22 cycles on a thermocycler and the PCR products were fractionated on a polyacrylamide gel. The DNA bands were stained with ethidium bromide and the gel was photographed with Kodak Tmax negative film under ultraviolet illumination.

Fig. 2B shows that the $\beta$-globin ( $460 \mathrm{bp} \mathrm{PCR}$ fragment) and c-fos ( $350 \mathrm{bp})$ bands were of similar intensity in undigested DNA (lanes 1). However, after extensive digestion with DNase I, the c-fos band became

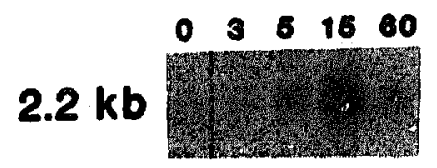

Fig. 1. Northern blot analysis of c-fos induction. Total RNA was purified from serum-starved HeLa cells induced with media plus $15 \%$ serum for $0,3,5,15$ or $60 \mathrm{~min}$. The RNA was then denatured by glyoxal, fractionated by agarose gel electrophoresis, transferred to nitroce!lulose and hybridized to the ${ }^{32} \mathrm{P}$-labeled PCR $\mathrm{c}$-fos probe shown in Fig. 2A.

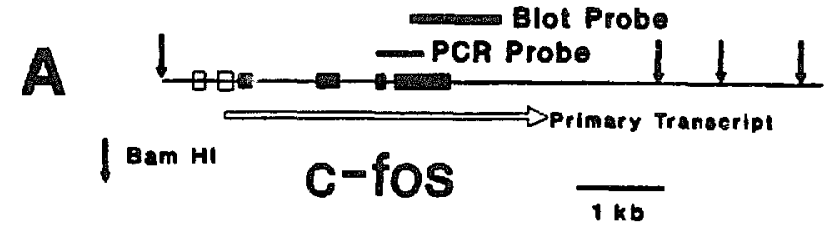

$\mathbf{B}$

0 min 15 min 60 min
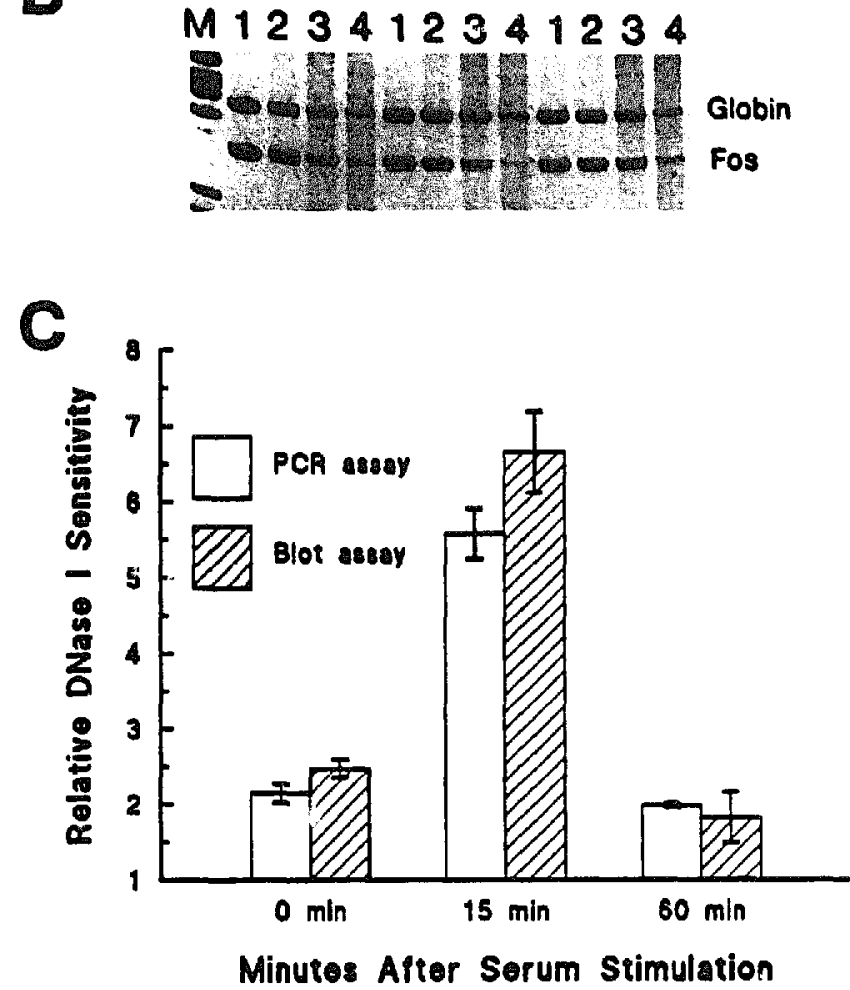

Fig. 2. Blot versus PCR assay of c-fos DNase I sensitivity. (A) Map of human c-fos. Coding regions are shown as solid boxes. The c-for enhancer (far left box) and promoter are depicted as open boxes. The blot probe (gel purified from cut plasmid DNA) was used to hybridize to a Southern blot containing DNase 1 digested samples and labels a 700 bp BamHl-Stul restriction fragment. The blot data was from our previous paper [5] and is not shown. The PCR probe consists of two complementary 30 base oligomers. which amplifies a 350 bp fragment (probe F2). (B) Negative photograph of DNase I.PCR gel. Nuclei were purified from serum-starved HeLa cells induced with $15 \%$ serum for 0,15 or $60 \mathrm{~min}$. The nuclei were digested with $0.0 .11,0.33$ or I $\mu \mathrm{g}$ of DNase I (lanes 1-4) and purified DNA was restricted with $P_{s /} I$. $\beta$-globin and c-fos primers were added along with $T a q$ polymerase to amplify 460 bp and 350 bp portions of the $\beta$-globin and $c$-fos genes, respectively. The amplified PCR products were electrophoresed on a $6 \%$ polyacrylamide gel. stained with ethidium bromide, and photographed in short ultraviolet light with negative film. (C) Southern blot (autoradiograph) and PCR (negative film) bands were quantitated using densitometry at 0 and $1 \mu \mathrm{g}$ DNase I (see Materials and Methods). Each determination denotes the mean \pm S.E. $(n=3)$.

relatively less intense compared to $\beta$-globin (lanes 4 ), especially at the 15 min time point. The band intensities from three separate assays were quantitated by densitometry and a histogram was prepared comparing the results of the PCR and the Southern blot assays. Fig. 2C demonstrates that the Southern blot and PCR 


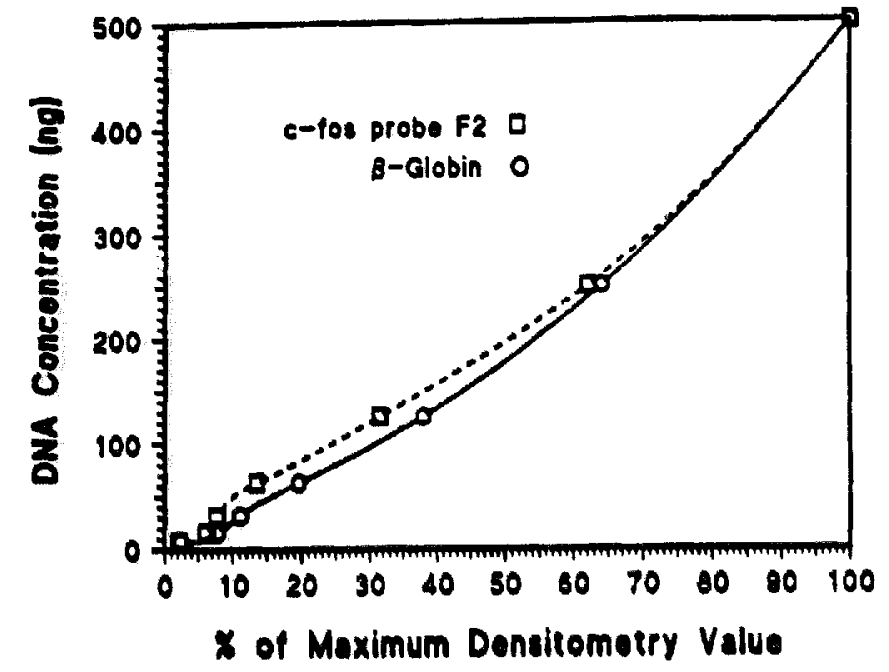

Fig. 3, PCR quantitation of diluted genomic DNA. Undigested HeLal DNA was restricted with $P s t l$ and then diluted by half in five separate dilutions. Each dilution was subjected to PCR amplitication using c-fos (probe F2) and $\beta$-globin primers. The products were visualized and quantitated by densitometry as in Fig. 2. ‥ c-fos probe $F_{2}: 0 . \beta \cdot g l o b i n$.

assays give similar relative DNase I sensitivities. However, the PCR assay is much faster, uses 20-fold less DNA and has a higher resolution.

The PCR method uses an exponential amplification in which the efficiency of amplification may increase with the decrease in target DNA concentration [15] (our unpublished findings). This variable efficiency has been one obstacle to making PCR a quantitative assay. Thus, the success of the PCR assay described above depends on careful quantitation using both internal and external standards [15-19]. In all of our c-fos assays we use the inactive $\beta$-globin genes as our internal standard by which the relative disappearance of c-fos is quantitated. As an external standard, we use dilution assays of genomic DNA to determine the PCR amplification efficiencies for each probe. An example of such a dilution assay for $\mathrm{c}$-fos and $\boldsymbol{\beta}$-globin is shown in Fig. 3. With the proper internal and external controls, we find that the PCR data obtained are simpler to analyze and of higher resolving power than any of the previous assays of DNA concentration using hybridization technology.

Enhancer-induced changes in DNase I sensiticity along the c-fos domain

To search for a gradient of DNase I sensitivity, we initially chose c-fos induction times of $5 \mathrm{~min}$ or less, since our early experiments indicated that the DNase I sensitivity gradient might be more pronounced during basal transcription or soon after serum induction. Fig. 4 shows a c-fos induction experiment in which serumstarved HeLa cells were stimulated with serum for 0 (circles), 3 (triangles) or 5 (stars) min. DNase I-PCR analysis was performed on the samples to determine relative sensitivities to DNase 1 digestion of the four downstream c-fos DNA regions (shown as dark lines

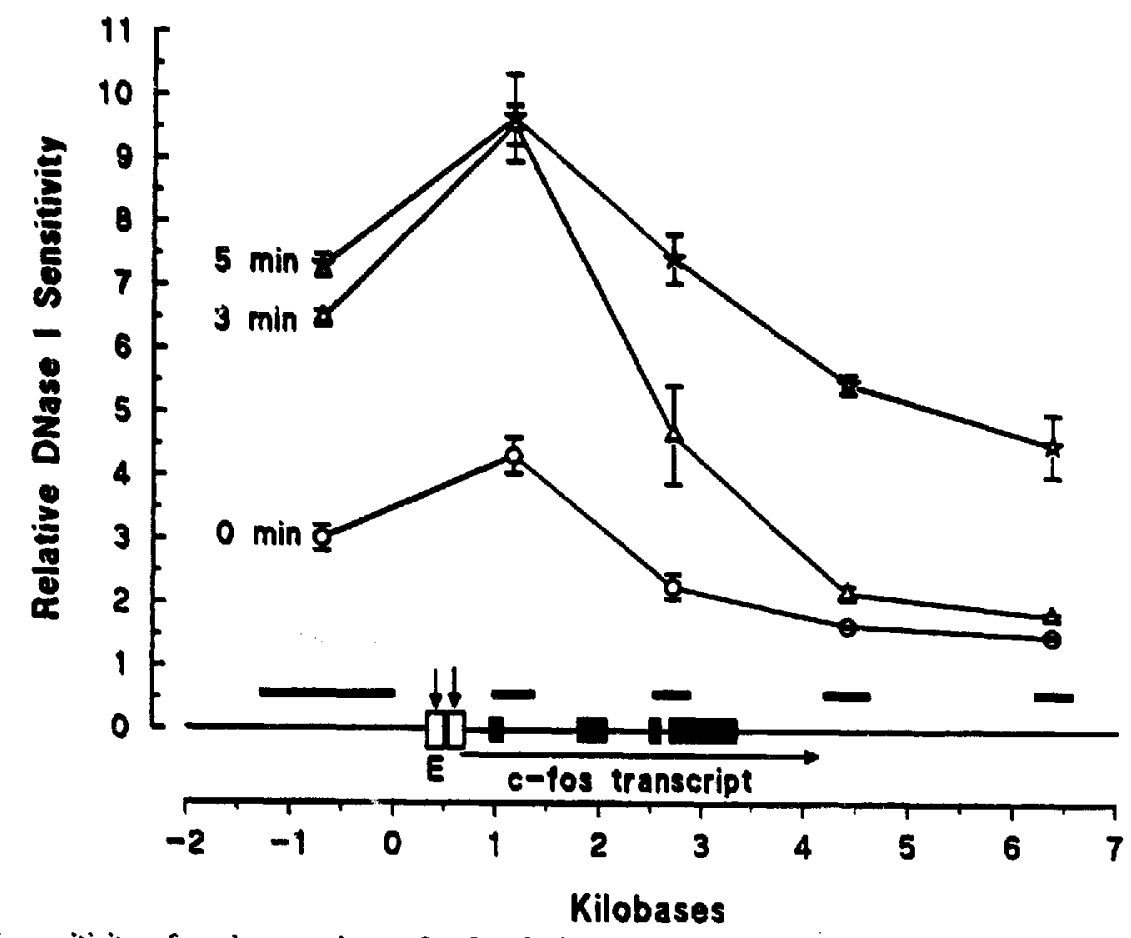

Fig. 4. Relative DNase I sensitivity of various regions of c-fos during the first $5 \mathrm{~min}$ of serum stimulation. Nuclei were isolated from serum-starved HeLa cells induced with 15\% serum for 0 (circies), 3 (triangles) or 5 (stars) min. The DNase I-PCR assay was performed as in Fig. 2 using the F1 through F4 c-fos PCR probes. The PCR probes are shown as black boxes above the c-fos map. The unsequenced upstream region was probed by the Southern blot assay as before [5]. The upstream probe is a $1.4 \mathrm{~kb}$ Eco RI-Bam HI restriction fragment and is shown as a black box above the $c$-fos map. Each determination denotes the mean \pm S.E. $(n=3)$. 


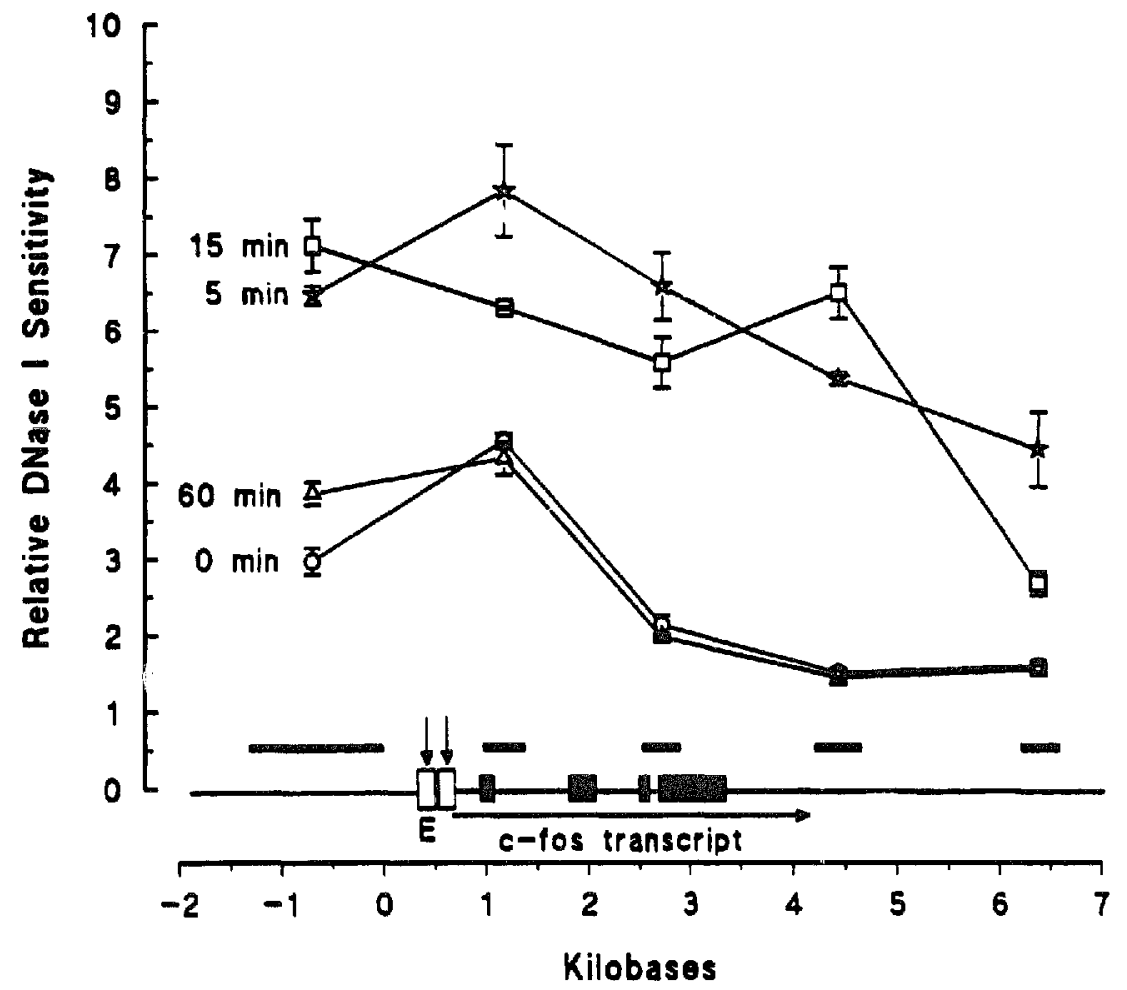

Fig. 5. Relative DNase I sensitivity of various regions of c-fos during the first 60 min following serum stinulation. Nuclei were isolated from serum-starved Hela cells induced with $15 \%$ serum for () (circles). 5 (stars). 15 (squares) or 60 (triangles) min. DNase I assays were performed as in Fig. 4. Each determination denotes the mean \pm S.E. $(n=3)$.

above the c-fos map). DNase I sensitivity of the unsequenced upstream region was determined by Southern blotting as before [5]. All data points for c-fos were normalized to $\beta$-globin and were corrected for target size. Fig. 4 demonstrates that before induction DNase l sensitivity is highest near the start-site of the gene and declines both upstream and downstream of the enhancer-promoter region. Thus, prior to stimulation of the c-fos enhancer, DNase I sensitivity peaks near the enhancer-promoter, with a $5^{\prime}$ to $3^{\prime}$ gradient of sensitivity appearing downstream.

Within the first 3 min of serum stimulation, DNase I sensitivities of the probes proximal to the enhancerpromoter increase preferentially, so that the $5^{\prime}$ to $3^{\prime}$ gradient of sensitivity is more pronounced (Fig. 4). However, after $5 \mathrm{~min}$ of serum stimulation, DNase I sensitivity is increased in the distal regions and chromatin accessibility appears more uniform across the c-fos domain (Figs. 4 and 5). We conclude that while serum stimulation initially enhances the gradient of c-fos DNase I sensitivity found in serum-starved cells. the effect is transient as chromatin accessibility soon spreads into the $3^{\prime}$ distal region with longer stimulation times.

Transcription of c-fos is detectable at low levels in the first 5 min following serum induction (Fig. 1 and Refs. 5,14). We wondered how DNase I sensitivity across the c-fos domain would change at $15 \mathrm{~min}$ when c-fos transcription peaks and at $60 \mathrm{~min}$ when transcrip- tion falls back to basal levels $[5,14]$. To investigate the longer stimulation times, serum-starved HeLa cells were stimulated with $15 \%$ serum for 0 (circles), 5 (stars), 15 (squares) or 60 (triangles) min. Nuclei were isolated and DNase 1 analysis was performed as before. Fig. 5 demonstrates that DNase 1 sensitivity remains high throughout most of the c-fos domain at $15 \mathrm{~min}$ and changes little from the $5 \mathrm{~min}$ time point. In contrast, at $60 \mathrm{~min}$ post-stimulation, DNase 1 sensitivity falls back to the low level found in serum-starved cells. As before, marked differences in DNase I sensitivity appear across the c-fos domain with the regions proximal to the enhancer-promoter having the highest accessibility.

\section{Discussion}

At low lecels of c-fos transcription, DNase I sensiticity peaks around the sequences proximal to the enhancerpromoter region

When HeLa cells are harvested after 0 or 60 min of serum stimulation, c-fos transcription is at basal levels (Fig. 1 and Refs. 5, 14). Under these conditions, Figs. 4 and 5 confirm our previous data that c-fos DNase I sensitivity declines at increasing distances from the promoter region. At $3 \mathrm{~min}$ of serum stimulation, a steeper gradient of DNase I sensitivity is generated. This is a transient state, however, as the gradient is reduced by $5 \mathrm{~min}$ after serum stimulation (Fig. 4). 
While c-fos mRNA levels increase from 5 to $15 \mathrm{~min}$ post-stimulation (Fig. 1 and Refs. 5, 14), the high steady-state transcription at $15 \mathrm{~min}$ does not lead to marked changes in DNase I sensitivity across the c-fos domain (Fig. 5).

While gradients of DNase 1 sensitivity have often been observed at the boundaries of DNase I sensitive domains [2-4], the gradient observed in the c-fos domain is novel because it declines within the c-fos transcription unit and because it is readily modulated by stimulation of the c-fos enhancer. An understanding of what generates these temporal and spatial patterns of DNase I sensitivity could help elucidate the significance of active DNase I sensitive chromatin in gene regulation.

The DNase I.PCR assay yields reliable high-resolution data on chromatin accessibility at any sequenced DNA locus

Since pancreatic DNase I is a non-specific DNA-binding protein, the DNase I assay has proven to be a valuable tool for mapping domains of chromatin accessibility around active gene regions. However, progress in understanding the functional significance of domain DNase I sensitivity has been disappointing. A good part of this lack of progress is due to the Southern blot, dot blot and solution hybridization assays previously available. Southern blots are disadvantaged because of the low resolution and the inconvenience of relying on appropriately placed restriction sites (comparisons of different sized restriction fragments can introduce large and unpredictable errors into serisitivity calculations). Dot blots and solution hybridization have somewhat better resolution, but often give spurious results due to nonspecific background hybridization. With such imprecise assays for DNase I sensitivity, only qualitative conclusions can be drawn.

In contrast to the DNase I assays based on hybridization technologies, the DNase I-PCR assay gives quantitative, high-resolution data on chromatin accessibility for any genomic locus using 20-fold less DNA, and is 10-fold faster. The PCR-based assay will allow the preparation of detailed maps of chromatin accessibility for any genomic region that has been sequenced and mapped for DNase I hypersens ive sites. The DNase I sensitivity data obtained are of high enough resolution to aid in elucidating the potential role of enhancers and other regulatory elements in modulating chromatin accessibility.

\section{Acknowledgments}

We thank Michael Pikaart and Harold Martinson for helpful criticisms. This work was supported by the National Science Foundation (DMB-9018701) and by BRSG 2 SO7 RR07050-25 awarded to the Office of the Vice President for Research, The University of Michigan by the Biomedical Research Support Grant Program, Division of Research Resources, National Institutes of Health.

\section{References}

I Stalder. J., Groudine, M., Dodgson, J.B., Engel, J.D. and Weintraub, H. (1980) Cell 19, 973-981.

2 Lawson, G.M., Knoll, B.J., March, C.J., Woo, S.L.C., Tsai, M.-J, and O'Malley, B.W. (1982) J. Biol. Chem. 257. 1501-1507.

3 Alevy, M.C., Tsai, M.-J. and O'Malley, B.W. (1984) Biochemistry 23. 2309-2314.

4 Jantzen, K., Fritton, H.P. and Igo-Kemenes, T. (1986) Nucleic Acids Res. 14, 6085-6099.

5 Feng, J.L. and Villeponteau, B. (1990) Mol. Cell. Biol. 10, 11261133.

6 Curran. T., Peter, S.G., Van Beveren, C., Teich, N.M. and Verma. I.M. (1982) J. Virol. 44, 674-682.

7 Curran, T., Miller, A.D., Zokas, L. and Verma, I.M. (1984) Cell 36, 259-268.

8 Beaudet, A.L., O'Brien, W.E., Bock. H.G.O., Freytag, S.O. and Su, T.-S. (1986) Adv. Hum. Genet. 15, 161-196.

9 Feng. J.. Irving. J. and Villeponteau, B. (1991) Biochemistry 30 , 4747-4752.

I0 Villeponteau, B. and Martinson, H. (1981) Nucleic Acids Res. 9. 3731-3746.

II Southern. E.M. (1975) J. Mol. Biol. 98, 503-517.

12 Chirgwin, J.M.. Przybyla, A.E., MacDonald, R.J. and Rutter, W.J. (1979) Biochemistry 18, 5294-5299.

13 McMaster, G.K. and Carmichael, G.G. (1977) Proc. Natl. Acad. Sci. USA $74,4835-4838$.

14 Greenberg, M.E. and Ziff, E.B. (1984) Nature 311, 433-438.

15 Becker-Andre, M. and Hahlbrock, K. (1989) Nucleic Acids Res. 17, 9437-9446.

16 Chelly, J., Kaplan, J.C., Maire, P., Gautron. S. and Kahn, A. (1988) Nature 333, 858-860.

17 Rappolee, D.A.. Mark, D.. Banda. M.J. and Werb, Z. (1988) Science 241, 708-712.

18 Wang, A.M., Doyle, M.V. and Mark, D.F. (1989) Proc. Natl. Acad. Sci. USA 86, 9717-9721.

19 Rupp, R.A.W. and Weintraub, H. (1991) Cell 65, 927-937. 